\title{
Les étudiants russes face à l'internationalisation de l’enseignement supérieur
}

\begin{abstract}
Avertissement
Le contenu de ce site relève de la législation belge sur la propriété intellectuelle et est la propriété exclusive de l'éditeur.

Les œuvres figurant sur ce site peuvent être consultées et reproduites sur un support papier ou numérique sous réserve qu'elles soient strictement réservées à un usage soit personnel, soit scientifique ou pédagogique excluant toute exploitation commerciale. La reproduction devra obligatoirement mentionner l'éditeur, le nom de la revue, l'auteur et la référence du document.

Toute autre reproduction est interdite sauf accord préalable de l'éditeur, en dehors des cas prévus par la législation en vigueur en Belgique.
\end{abstract}

\section{Référence électronique}

Citation : Oxana Gilyuk, «Les étudiants russes face à l'internationalisation de l'enseignement supérieur », Émulations, en ligne. Mise en ligne le 20 octobre 2016. URL : http://www.revue-emulations.net/enligne/etudiants-russesinternationalisation-enseignement-superieur-gilyuk

Éditeur : Émulations - Revue des jeunes chercheuses et chercheurs en sciences sociales http://www.revue-emulations.net 


\title{
Les étudiants russes face à l'internationalisation de l'enseignement supérieur
}

\author{
Oxana Gilyuk \\ Doctorante en sociologie à l'École des hautes études en \\ sciences sociales (Paris), Centre Maurice Halbwachs
}

\section{Résumé/Abstract}

[Fr] Cet article montre que les compétences linguistiques sont inégalement distribuées en Russie contemporaine. La probabilité de devenir mobile internationalement varie alors pour les étudiants russes. De même, ce n'est pas avec une même temporalité ni avec une même légitimité que ces derniers envisagent un voyage d'études à l'étranger. Ces variations sont en lien avec les ressources socio-économiques et culturelles inégales de leurs familles d'origine.

\begin{abstract}
Mots clés : migration étudiante, Russie, inégalités sociales, socialisation, mobilité sociale.
[En] This article shows that linguistic competencies are unequally distributed in today's Russia. The probability of becoming internationally mobile then varies for Russian students. Equally, it is not with the same time constraints, nor with the same legitimacy that they are considering a study trip abroad. These variations are linked to the disparate socio-economic and cultural resources of their families.
\end{abstract}

Keywords: student migration, Russia, social inequality, socialization, social mobility.

\section{Introduction}

Avec la disparition de l'URSS, la possibilité s'ouvre pour les jeunes Russes d'effectuer un voyage d'études à l'étranger. Les visas de sortie sont supprimés, ce qui facilite la circulation internationale des citoyens russes, tandis que l'enseignement des langues étrangères connaît un essor dans la Russie des années 1990. À cela s'ajoute l'internationalisation croissante de l'enseignement supérieur dans le monde à partir de ces mêmes années. C'est ainsi que les étudiants russes apparaissent sur la scène internationale, souhaitant étudier, à l'étranger, le commerce, la gestion, les sciences sociales ou les arts. Le nombre d'étudiants russes qui reçoivent une formation supérieure à l'étranger a doublé en quelques années, passant de 25946 en 1999 à 51171 en 2012 ${ }^{1}$. L'Allemagne, les États-Unis, la France et la Grande-Bretagne constituent le peloton de tête de leurs pays d'accueil ${ }^{2}$.

Ce constat ne masque toutefois pas un autre : la majorité des étudiants russes ne sont pas mobiles internationalement. Selon Campus France, les Russes qui reçoivent une formation à l'étranger représentent, en 2009 , moins de $0,5 \%$ du total des étudiants de ce pays, et ce chiffre n'évolue quasiment pas pour l'année $2012^{3}$. Les enquêtes nationales disponibles attestent simultanément que nombreux sont, en Russie, les étudiants qui souhaiteraient poursuivre leur éducation à l'étranger : $45 \%$ pour ne citer que le chiffre le plus modeste ${ }^{4}$. La question se pose

1 « Russie », Fiches pays Campus France, novembre 2014. En ligne. URL : http://www.campusfrance.org/fr/ressource/russie-2013-2014. ${ }^{2}$ Ibid.

3 " Russie », Dossiers de Campus France, $\mathrm{n}^{\circ} 22$ septembre 2014. En ligne. URL : http://ressources.campusfrance.org/publi_institu/agence_cf/dossiers/fr/dossier_22_fr.pdf.

${ }^{4}$ Les résultats du sondage effectué en Russie en mai 2013 par le Centre analytique Levada sont consultables en ligne. URL : http://www.levada.ru/06-06-2013/mechty-ob-emigratsii. Tout en soulignant le déficit de recherches en matière de mobilité d'étudiants russes, effective comme aspirée, on pourrait également évoquer d'autres enquêtes de taille et de crédibilité variables dans lesquelles ce chiffre grimpe vers 53,6\% (URL : http://www.examen.ru/add/previous-polls/Opros-Obucheie-Za-Granitseisondage), voire vers $80 \%$ (URL : http://www.study.ru/support/lib/note77.html). 
sur les raisons du choix de la mobilité ainsi que sur celles de l'écart si important entre les deux taux : $0,5 \%$ seulement de ces $45 \%$ parviennent à effectuer un séjour d'études à l'étranger. Quels sont ces jeunes qui souhaiteraient étudier à l'international ? Quels obstacles rencontrentils à la réalisation de leur souhait? Les contraintes auxquelles ils font face sont-elles communes à tous les étudiants russes ou plutôt propres à certains groupes au sein de cette population ? En s'appuyant sur les matériaux recueillis dans le cadre d'une thèse de doctorat, cet article voudrait apporter des éléments de réponses à ces questions.

La confrontation des entretiens auprès des jeunes Russes mobiles à l'international avec ceux menés auprès de leurs homologues non mobiles a fait immédiatement apparaitre leur hétérogénéité en termes de milieu d'origine et de ressources scolaires. Les données ne sont toutefois pas univoques. D'une part, le corpus d'entretiens conduits avec les mobiles comporte plus d'enfants de parents hautement diplômés et aisés. De même, on y dénombre davantage d'anciens élèves d'écoles renforcées en langues étrangères ou d'autres écoles élitistes. Ce constat établi sur le terrain russe converge avec les résultats de plusieurs enquêtes sur la mobilité étudiante. Ces dernières montrent que l'accès à des études à l'étranger est inégal selon les catégories socio-économiques des étudiants, que ceux-ci soient des Européens encadrés par Erasmus (Ballatore, 2010), des étudiants chinois (Yan, 2009), marocains (Gérard, 2008) ou québécois (Garneau, 2008). En particulier, ce sont les compétences linguistiques qui varient au sein d'une société nationale. Comme le suggère Wagner (2007), la maîtrise des langues étrangères définit une des formes du capital distinctif des classes supérieures ou moyennes fortement scolarisées, ces dernières étant surreprésentées parmi les étudiants internationaux.

D'autre part, l'hétérogénéité des interviewés en termes de ressources socio-économiques et scolaires ne couvre pas parfaitement la distinction entre les mobiles et les non mobiles. En effet, certains, à l'intérieur des deux corpus, peuvent être décrits comme appartenant à une «jeunesse dominante », scolairement et économiquement. Leur trajectoire est marquée par l'accumulation, précoce et coûteuse, de compétences linguistiques, même si ce n'est pas dans tous les cas qu'une mobilité d'études se produit pour ce groupe. D'autres interviewés, mobiles comme non mobiles, sont clairement issus de milieux plus modestes et leur scolarité apparaît comme moins compétitive. En disposant certes de moins d'atouts pour rejoindre l'enseignement supérieur à l'étranger, nombre d'entre eux y parviennent, parfois explicitement sur le mode d'un « destin d'exception », à l'instar de certains étudiants français qui ont accédé à l'université dans leur pays dans les années 1960 (Bourdieu, Passeron, 1964 : 42). Ce constat amène à reconnaître les limites de l'approche en termes de variables. Sans nier la pertinence ni les apports de cette dernière, on se propose de la compléter par celle qui vise à restituer les processus par lesquels les étudiants, inégalement dotés en compétences et ressources, conçoivent et réalisent - ou non - un voyage d'études hors Russie.

Il s'agit, dans cet article, de montrer que l'environnement familial et scolaire influence la probabilité de devenir mobile à l'international, ainsi que les modalités de la conception d'un voyage d'études. Dans un premier temps, nous verrons qu'apprendre des langues étrangères dans la Russie contemporaine est à mettre en lien avec les ressources socio-économiques et culturelles des familles d'origine. Une partie des jeunes Russes s'avère ainsi être mise précocement à l'écart de la possibilité d'envisager confortablement une mobilité internationale d'études, voire d'y songer. Dans un second temps, nous observerons de quelles manières les expériences socialisatrices familiales et scolaires variables des interviewés autorisent à concevoir une mobilité. Si certains interviewés envisagent aisément et précocement un voyage d'études à l'étranger, pour d'autres, une possibilité de devenir mobiles internationalement ne s'ouvre que tardivement. Cette possibilité est accompagnée du rattrapage hâtif des compétences linguistiques et s'effectue au prix d'une rupture avec leur milieu d'origine et leurs représentations, acquises précédemment, au sujet d'une éventuelle mobilité étudiante.

\section{Précisions sur l'enquête}

Cet article s'inscrit dans un travail de thèse en cours sur la mobilité d'études des Russes vers la France. Il s'agit de repérer les conditions qui les amènent à concevoir la mobilité, ainsi que les actions qu'ils effectuent pour la mettre en œuvre. 
L'analyse s'appuie sur 100 entretiens biographiques qui forment deux corpus. Le premier comporte 70 entretiens (45 femmes et 25 hommes) menés en France (Paris, Lyon, Marseille, Metz) auprès de jeunes en mobilité internationale. Ceux-ci, nés principalement au cours des années 1980/début 1990, sont issus de milieux sociaux variés et originaires de différentes villes de l'ancienne URSS. Ils sont étudiants ou diplômés d'établissements d'études supérieures français (65 cas) ou d'autres pays occidentaux (5 cas), publics ou privés, proposant des formations diverses, allant du management aux arts plastiques. Ce groupe compte des cas de mobilité dite spontanée et autofinancée, ainsi que de mobilité effectuée dans un cadre institutionnel et/ou avec une bourse d'études.

Pour mieux saisir les conditions favorisant ou décourageant la mobilité internationale étudiante, le second corpus de 30 entretiens ( 16 femmes et 14 hommes) a été réalisé en Russie auprès de jeunes «non mobiles » qui n'ont pas connu, au moment de l'entretien, d'expérience d'études à l'étranger. Ce sont des étudiants ou des diplômés d'établissements supérieurs de Moscou et de Saint-Pétersbourg, ainsi que de villes de province (Ekaterinbourg, Novossibirsk et Irkoutsk). Ils ont sensiblement le même âge que les interviewés en mobilité, sont parfois leurs amis ou connaissances (12 cas), leurs frères ou leurs sœurs (3 cas). Hormis le critère d'âge, une diversité de filières et de lieux d'études a été recherchée.

Tous les entretiens ont été conduits en russe. Ils portaient sur les parcours de vie (milieu familial, passé scolaire et choix d'études supérieures), les aspirations et les représentations des interviewés concernant la mobilité étudiante. Vers la fin de chaque entretien avec les non mobiles, la question sur un départ éventuel à l'étranger a été posée de façon plus ou moins fluide selon les cas. La recherche était présentée à ce groupe d'interviewés comme visant à étudier les parcours des jeunes générations.

Deux concepts théoriques ont été retenus pour analyser le matériau recueilli. Il s'agit, d'une part, de considérer la mobilité étudiante en question comme produit d'une socialisation, c'est-à-dire d'un processus socialement différencié par lequel se forment les membres d'une société nationale à une époque historique concrète (Darmon, 2012). En particulier, les socialisations familiales et scolaires variables des interviewés se révèlent contribuer différemment à l'émergence d'une future mobilité. On propose, d'autre part, de traiter la mobilité d'études comme une carrière faite d'étapes (Becker, 1985 ; Darmon, 2008). Il est notamment apparu pertinent de distinguer deux phases dans le début de la carrière de mobilité. Durant la première phase, celle de la conception d'un voyage d'études à l'étranger, la personne se fait une idée de son éventuel départ, sans dépasser pour autant le simple souhait. Ce dernier se réalise durant la seconde phase, celle de la préparation du départ. Le candidat à la mobilité y effectue des démarches pour faire certifier ses compétences linguistiques, parfois apprendre une nouvelle langue étrangère, s'inscrire dans un établissement d'études et obtenir le visa étudiant, le cas échéant une bourse. Cet article va principalement traiter de la première phase de la carrière de mobilité et montrer que les variations dans son déroulement sont à mettre en lien avec l'environnement familial et scolaire des interviewés. Notons à ce propos que, malgré un nombre important de travaux sur les étudiants internationaux (Endrizzi, 2010), peu d'attention est accordée aux modalités concrètes des processus - qui ne sont pas exactement les mêmes pour tous - par lesquels ces étudiants conçoivent et préparent leur voyage d'études à l'étranger.

\section{Conditions inégales d'acquisition des compétences en langues étrangères}

Il ressort des entretiens que les compétences linguistiques des interviewés, mobiles comme non mobiles, sont variables à la sortie du secondaire. Un «talent » pour les langues étrangères est souvent évoqué dans les entretiens comme un fondement de leur maîtrise. Certains interviewés se plaignent ainsi de l'absence dudit « talent» et se qualifient de «mauvais en langues », alors que d'autres se considèrent « doués » en la matière. La production des performances en langues étrangères doit néanmoins davantage à des conditions sociales permettant d'acquérir une compétence linguistique qu'à des « dons » individuels.

Les écarts entre les interviewés renvoient en particulier à leurs inégalités de scolarisation reflétant celles socio-économiques et culturelles de leur famille d'origine. 


\subsection{L'idéal égalitaire mis à mal : l'enseignement secondaire de Russie en recomposition}

Sans prétendre à la description complète de l'historique du système d'enseignement secondaire en Russie, il convient d'évoquer ici ses quelques spécificités.

Les discussions sur la création d'un nouveau système scolaire débutent en URSS à la prise du pouvoir par Mikhaill Gorbatchev. Les débats se poursuivent et prennent la forme de lois réformatrices dans les années 1990. Les réformes mises en place traduisent le souhait de se départir de l'école soviétique - qualifiée de rigide, excessivement centralisée et égalitaire - et de créer un nouveau système scolaire correspondant à la société ouverte et démocratique (Berelowitch, 2000). L'adoption, en 1992, de la loi «Sur l'enseignement » de la Fédération de Russie introduit une véritable diversification de l'enseignement secondaire dans le pays. Les écoles acquièrent désormais une autonomie importante dans l'élaboration des programmes d'enseignement et dans le mode d'admission des élèves ; ces derniers ont, quant à eux, le droit de choisir leur établissement. L'ouverture d'écoles privées est également consacrée par cette loi, ainsi que les transferts des ressources extrabudgétaires, dont celles des parents d'élèves, dans les écoles publiques, via l'introduction de classes ou de disciplines payantes (Vinokur, 2001). On observe, en parallèle, la réduction importante du financement public de l'Éducation nationale (de plus de $40 \%$ entre 1990 et 1997), les arriérés de salaires des enseignants et la baisse de leur valeur réelle (Vinokur, 2001). Du surcroît, des crises politiques et économiques majeures ainsi qu'un important reclassement social qui accompagnent la dissolution de l'Union soviétique aggravent les inégalités socio-économiques dans le pays (Rey, 2005). L'inflation, la décroissance industrielle et les impayés des salaires dans le secteur public provoquent une dégradation du niveau de vie pour une grande partie de la population russe. Pour une autre partie, la privatisation et l'instauration de l'économie de marché permettent la prospérité et l'édification de fortunes parfois colossales. La politique en matière d'éducation ne fait qu'accroître l'ampleur des inégalités. Elle contribue notamment, sous une apparence parfaitement légitime de la démocratisation de l'enseignement secondaire, à la différenciation prématurée de cursus scolaires de jeunes générations russes à l'intérieur desquelles « les privilèges sont accordés à ceux qui bénéficient au départ d'avantages socio-économiques et culturels » (Tcherednitchenko, Lapparent, 2000 : 107). Étrangement, ce sujet est loin d'être au centre de l'intérêt des chercheurs travaillant sur la Russie, et peu d'analyses empiriques sont consacrées aux tenants et aboutissants de la différenciation scolaire précoce mise en place dans ce pays durant les années 1990.

Par ailleurs, les réformes évoquées ne partent pas d'une page blanche. En particulier, deux centaines d'écoles "spécialisées » (appelées sous forme abrégée «écoles spéc »), en langues étrangères et en mathématiques, sont créées en URSS dans les années 1960, principalement à Moscou et à Leningrad (Tcherednitchenko, 1993). Réservées aux enfants de l'establishment national, elles témoignent de la distinction sociale au sein du système scolaire dès l'époque soviétique. Depuis les années 1990, s'élargit et se complexifie ainsi un système d'établissements d'enseignement secondaire à deux vitesses. Y coexistent, d'une part, les écoles élitistes (anciennes comme nouvellement créées «écoles spéc », ainsi que « gymnasias » et "lycées ", labels d'apparition plus récente et pratiquant la répartition des enfants dans des classes d'options), effectuant une sélection des élèves à l'entrée et proposant un enseignement approfondi dans une ou plusieurs matières ainsi qu'une préparation à des filières sélectives postscolaires, et, d'autre part, les écoles secondaires « de masse » (ou « ordinaires », pour reprendre les expressions utilisées en Russie) qui ne dispensent qu'un enseignement de base et souffrent de fluctuation de personnel, d'obsolescence du matériel et de classes surchargées (Tcherednitchenko, Lapparent, 2000).

Durant l'année 1998-1999, 12 \% environ des élèves russes fréquentent une école privilégiée (Vinokur, 2001 : 114), et cette proportion est significativement plus élevée chez mes interviewés qui étaient, durant cette même période, élèves d'une école secondaire. Ainsi, parmi 70 interviewés en mobilité internationale d'études, 44 (donc $63 \%$ ) sont d'anciens élèves d'écoles privilégiées, dont 25 (36\%) ont fréquenté une école renforcée en langues étrangères et $19(27 \%)$ une autre école «spécialisée », notamment renforcée en mathématiques. Parmi les 
non mobiles, on trouve 13 anciens élèves d'écoles privilégiées sur 30 interviewés (donc $43 \%$ ), dont $3(10 \%)$ sont d'anciens élèves d'une école renforcée en langues et $10(33 \%)$ d'une autre école privilégiée.

Concernant la compétence en langues étrangères, les différences sont patentes entre les interviewés qui ont fréquenté une école de masse ou une version élitiste. S'agissant d'éventail des langues proposées, d'horaires et méthodes de l'enseignement, ou de qualification des enseignants, les anciens élèves d'écoles spécialisées avaient considérablement plus de chances d'acquérir des compétences solides en plusieurs langues étrangères que les anciens élèves d'écoles ordinaires. Ces derniers fournissent surtout des informations sur la pauvreté des résultats de l'apprentissage linguistique dans leur école : ils sont nombreux à en sortir et à déclarer ne connaître au mieux que des rudiments d'une seule langue étrangère. De même, selon certains, les langues étrangères, tout comme d'autres matières scolaires, n'ont pas été enseignées de façon régulière dans leur école, faute de professeurs.

La quasi-assurance de l'entrée à l'université peut être associée aux écoles privilégiées, les inégalités de l'éducation supérieure ne faisant que prolonger celles dans le secondaire. Les statistiques (Tcherednitchenko, Lapparent, 2000), comme les témoignages de mes interviewés, attestent que la quasi-totalité des anciens élèves d'écoles privilégiées deviennent étudiants en supérieur et qu'un nombre important parmi eux intègre les universités les plus prestigieuses du pays. Parmi ces dernières, figurent l'Université d'État de Moscou, l'Université d'État de SaintPétersbourg, l'Université de relations internationales de Moscou et l'École des hautes études en économie de Moscou. Ces établissements entretiennent des relations étroites avec des partenaires étrangers et proposent des cursus internationaux intégrés. Quant aux anciens élèves d'écoles de masse, ils entrent moins souvent à l'université et accèdent plus fréquemment à des filières supérieures moins prestigieuses, voire de relégation, rarement touchées par la coopération internationale entre universités.

\subsection{Les filtres d'entrée dans les écoles réputées}

Comme 1'exprime Diana ${ }^{5}$ (mobile) ayant fréquenté une école renforcée en français, une référence de l'enseignement $\mathrm{du}$ français à Saint-Pétersbourg, l'entrée dans de tels établissements n'est pas accessible aux « simples mortels ». En effet, inscrire l'enfant dans une école réputée, susceptible de délivrer un enseignement secondaire d'une meilleure qualité dans une ou plusieurs disciplines, n'est pas à la portée de toutes les familles, même s'il s'agit par excellence d'écoles publiques ${ }^{6}$.

Les inscriptions dans les écoles réputées apparaissent d'abord prédéterminées par la localisation spatiale de ces dernières (principalement les centres de grandes villes avec la plus haute concentration à Moscou et à Saint-Pétersbourg). Les interviewés ne sont ainsi pas égaux devant l'offre de l'enseignement linguistique en fonction de leur lieu de résidence, ceci étant souvent une répercussion des caractéristiques socio-économiques de leur famille d'origine. Par exemple, selon Diamara (mobile), fille de petit commerçant, la plupart de ses camarades de classe d'une école linguistique du centre de Moscou appartenaient à « la crème de la crème »plusieurs étaient des enfants de célébrités qui habitaient à proximité. Ceux-là sont tous entrés à l'université nationale. Selon Tamara (mobile), fille de machiniste de locomotive, scolarisée dans une école ordinaire d'une petite ville industrielle de l'Oural, la majorité de ses camarades de classe constituait «les enfants d'ouvriers ». Seulement trois parmi eux ont intégré des études supérieures en Russie.

Si l'admission dans les écoles spécialisées ne s'effectue pas nécessairement en fonction du lieu de résidence, tous les parents ne peuvent néanmoins pas se permettre d'inscrire leur enfant dans un établissement sélectif. En termes de recrutement des élèves, les entretiens informent

\footnotetext{
${ }^{5}$ Les prénoms des interviewés ont été modifiés. Afin d'éviter les répétitions, certains pseudonymes sont suivis d'une lettre indiquant la lettre initiale d'un nom de famille, qui est aussi fictif (i.e. Marina P.).

${ }^{6}$ Seule une personne parmi mes interviewés a été scolarisée dans une école privée destinée, pour reprendre ses propos, aux « enfants d'avocats ou d'autres nouveaux riches ». Il s'agit d'Irina (mobile) qui bénéficie de l'inscription gratuite en tant qu'enfant d'une des enseignantes de cette école.
} 
sur trois filtres d'entrée dans les écoles réputées : le sponsoring parental envers l'école, les réseaux de relations des parents et le concours d'entrée.

Il peut s'agir d'un don parental à l'école. Ainsi, pour inscrire Rodion (non mobile) dans une école anglaise de Saint-Pétersbourg, son père, manager d'une compagnie russo-britannique, a effectué «un don officiel de bienfaisance », comme le dit l'interviewé, en offrant une vingtaine d'ordinateurs pour la classe informatique. L'école peut aussi faire payer les parents, de manière déguisée, durant toute la scolarité, comme en témoigne Élisaveta (mobile), dont la mère est propriétaire d'une galerie d'art à Saint-Pétersbourg. Les parents d'élèves de son école («une des meilleures écoles de la ville, pour ne pas dire la meilleure ») ont été régulièrement amenés à payer la restauration des façades du bâtiment, la rénovation du parquet ou l'aménagement de la salle de motricité. L'interviewée évoque aussi l'existence de "classes $E$ » destinées aux élèves dont les parents payaient «officiellement» leur scolarité dans cet établissement public.

Au-delà de taxes d'entrée ou d'enseignement, inscrire un enfant dans une école spécialisée peut être conditionné par les réseaux de relations des parents. Quelques interviewés en parlent, parfois de façon euphémique. On comprend que tous les parents ne peuvent pas entretenir des relations de proximité avec le directeur d'établissement ou avec une autre personne influençant la décision d'admission. Seuls le peuvent les parents occupant une position socioprofessionnelle élevée, mais aussi parfois des enseignants (deux cas) ou des anciens élèves (quatre cas) de cette même école.

Enfin, le concours d'entrée représente une forme indirecte de sélection en fonction des ressources socioculturelles et/ou économiques des familles. Comme il ressort des récits, ce sont les parents diplômés qui cherchent le plus souvent un cadre scolaire particulier pour leurs enfants. Quelques interviewés affirment également avoir suivi, entre leurs six et sept ans, des « cours préparatoires » payants que certaines écoles réputées proposent aux familles de futurs élèves. Cette modalité d'inégalité d'accès à une école spécialisée est par ailleurs fortement naturalisée par les interviewés, notamment par ceux qui sont issus de familles d'élites intellectuelles (chercheurs, enseignants,...), économiquement déstabilisées durant les années 1990. En stigmatisant communément la sélection qui s'effectue selon les revenus des familles, ils ne trouvent pas injuste l'admission par concours qui vise des élèves « doués » et « intelligents ».

Les parents diplômés se révèlent également plus préoccupés par la réussite scolaire, sinon sociale, de leurs enfants. Celle-ci est clairement associée, dans la Russie des années 1990, aux compétences linguistiques développées. C'est le cas de Vadim (non mobile), par exemple, dont les parents, chefs d'un bureau privé d'architecture en province, l'inscrivent à des cours privés de français en dehors de la «gymnasia » que cet interviewé fréquente. En effet, dans cet établissement, l'enseignement de la deuxième langue étrangère ne démarre que dans les classes terminales. Ce pouvoir d'acheter une offre scolaire manquante ou de meilleure qualité ne peut pas, par ailleurs, être attribué aux milieux peu aisés. Pour comparer, les parents peu diplômés, même habitant de grandes villes, sont davantage en position de non-demande par rapport à l'offre existante en enseignement linguistique pour les enfants. Le cas de Stépan (non mobile) le prouve, fils de chauffeur de bus habitant à Saint-Pétersbourg.

En outre, l'existence d'un classement interne des " écoles spéc » s'entrevoit à travers des entretiens, notamment en fonction de la place des langues enseignées sur le marché des langues. En effet, à l'heure de la massification de la langue anglaise, le français est perçu en Russie comme une langue étrangère accessoire, et non pas comme la langue clé à l'international (Markosyan, 2000). L'accès à une école française ou à une classe française au sein d'une école linguistique devient du fait moins sélectif. Comme le confie Zhanna (mobile), ancienne élève d'une école française, ses parents, pétersbourgeois, dotés de moyens financiers modestes, ont examiné trois écoles publiques du quartier. La première a été qualifiée par eux de «la pire », la deuxième de «trop céleste » (trop difficile d'y accéder) et, enfin, la troisième, une école renforcée en français, celle qui a été choisie, de « solution moyenne ».

Pour résumer cette partie, la brève analyse des conditions d'accès à une éducation linguistique scolaire de qualité montre que la possibilité d'apprendre précocement une langue étrangère et, parfois, le choix de telle ou telle langue comme première langue étrangère renvoient avant tout aux ressources socio-économiques et culturelles des familles d'origine des interviewés. 
D'autres variables, comme le genre ou la période de la scolarisation, peuvent être évoquées ici de façon rapide en tant que contribution à l'explication des inégalités de l'éducation linguistique reçue par les personnes rencontrées. Les hommes interviewés sont ainsi plus nombreux à se déclarer peu familiers avec les langues étrangères à la sortie du secondaire que les femmes. Nous sommes face à un reflet d'un «profilage sexué » (Duru-Bellat, Jarlégan, 2001 : 86) des enfants au sein de l'école secondaire où les compétences linguistiques, tout comme celles en littérature ou en sciences humaines, sont perçues davantage comme un attribut féminin. De même, en comparant les cas qui s'approchent du modèle « toutes choses égales par ailleurs », dont ceux au sein d'une même fratrie, on constate que les interviewés nés autour des années 1990-1992 sont plus familiers avec les langues étrangères que leurs aînés de quelques années. Ces premiers ont plus d'opportunités d'apprendre les langues dans le cadre scolaire marqué, à partir des années 1990, par l'augmentation de l'utilité sociale des compétences linguistiques sur le marché d'emploi et modifiant ses programmes en conséquence. De même, cette acquisition est favorisée par les voyages et l'évolution de l'environnement médiatique et informatique en Russie.

Nous verrons dans la partie suivante que les modalités de l'éducation linguistique reçue par les interviewés durant leur scolarisation en Russie influent sur leur façon de concevoir un voyage d'études à l'étranger.

\section{Variations dans la conception d'une mobilité internationale d'études}

Au moins deux axes de variations sont repérables dans le déroulement de la première phase de la carrière de mobilité où une aspiration se forme quant aux études à l'étranger. Ces variations sont relatives à la temporalité de la construction d'un souhait d'aller à l'étranger et à la légitimité que les interviewés attribuent à celui-ci. Elles reflètent les expériences socialisatrices familiales et scolaires que connaissent les interviewés issus de groupes socio-économiques variés.

\subsection{Un souhait plus ou moins ancien d'étudier à l'étranger}

Pour une partie des interviewés mobiles, un voyage d'études à l'étranger se conçoit depuis longtemps, et l'entourage familial, puis scolaire et universitaire, y participe amplement. Bien qu'ils ne puissent pas être associés aux «héritiers de la culture internationale » élevés dans des environnements multilingues depuis leur jeune âge (Wagner, 2007), ces interviewés sont les plus dotés, parmi les personnes rencontrées, en compétences linguistiques. Ils invoquent fréquemment un «depuis toujours » pour parler de leur mobilité, présentée comme allant de soi : «Je voulais partir depuis l'enfance. [...] Depuis toujours, j'en avais envie. Une idée fixe, quoi » nous communique Alina, ancienne élève d'une école anglaise.

$\mathrm{La}$ famille et les institutions scolaires se révèlent être dans leurs cas deux institutions socialisatrices complémentaires. Ces interviewés proviennent des milieux les plus favorisés, économiquement, socialement et scolairement au sein de la population de l'enquête. Leurs parents sont hautement diplômés ; ils exercent souvent des activités professionnelles développées à l'international (ingénieurs et managers de compagnies internationales, traducteurs-interprètes, diplomates). Certains de ces parents sont présentés dans les entretiens comme disposés à une émigration; c'est le cas, par exemple, de ceux de Natalia qui possèdent à Moscou un «business » lié à l'audit et au leasing financier et, pour reprendre les propos de l'interviewée, "réfléchissent depuis longtemps à la manière de se déplacer à Londres ». Ces parents sont souvent caractérisés par les interviewés comme "pro-occidentaux et russophobes » (Oleg). Ils contribuent largement à la conception d'une mobilité internationale chez leurs enfants, en prenant part à la formation de leurs opinions sur la Russie et d'autres pays, en les initiant à des voyages à l'étranger et en prêtant attention à leur éducation linguistique. Ces mêmes parents apparaissent souvent par la suite comme les financeurs principaux, voire uniques, du séjour d'études de leurs enfants qui sont susceptibles de choisir les cursus internationaux les plus sélectifs et coûteux, rarement couverts par un financement institutionnel.

Une importance particulière a été accordée à l'enseignement précoce des langues étrangères pour ces interviewés, tous anciens élèves des écoles les plus réputées de Russie, de la part de 
leurs parents. Dans les cas les plus aboutis, l'école se présente comme une sorte de fabrique collective où, en s'influençant mutuellement, les élèves apprennent à envisager la mobilité internationale comme un avenir tout à fait probable. Comme l'explique Varvara, ancienne élève d'une école spécialisée en français, «partir étudier en France n'était globalement pas quelque chose d'extraordinaire » dans son milieu scolaire : «nous grandissions à peu près tous avec l'idée que nous partirions ensuite en France ».

Un nombre important de voyages touristiques et de séjours linguistiques à l'étranger durant les années scolaires, puis universitaires, caractérise ce groupe dont l'éducation linguistique est rarement strictement scolaire. Quelques-uns effectuent une partie de leurs études secondaires (d'une à trois années) à l'étranger : dans un lycée français conventionné à Sofia pour Olga D., dans une école internationale anglophone pour Natalia et dans une école américaine pour Rada.

Cette ouverture préalable à l'international se poursuit pour plusieurs pendant leurs études supérieures en Russie, dans les universités les plus renommées possédant des programmes d'échange en vigueur, et dans les disciplines tournées vers l'international (management, économie, relations internationales,...). Leur engagement dans le départ étudiant constitue, en effet, un acte de conformité à la logique de leur milieu universitaire, et on observe une continuité des milieux socialisateurs dans lesquels ces interviewés évoluent depuis leur jeune âge.

Dans d'autres entretiens menés auprès des interviewés mobiles, on constate une situation toute différente. Il n'existe pas de longue période de mûrissement de l'idée provenant des pratiques et des interactions en famille et à l'école ni de construction progressive des compétences et de l'expérience qui viendraient offrir un stimulus pour un éventuel départ étudiant. À la différence des cas précédents, où le départ à l'étranger est présenté comme réfléchi " depuis toujours », les interviewés de ce groupe disent n'y avoir jamais pensé. Ce « jamais » apparait de façon parfois trop insistante, comme lors de l'entretien avec Anton qui tient à souligner à plusieurs reprises combien son voyage d'études était inédit pour lui et pour son entourage :

Jamais personne de ma famille n'était allé à l'étranger. [...] Jamais je n'ai pensé, moi, faire mes études à l'étranger. [...] Je n'ai jamais été calé pour les langues. [...] Jamais la France ne me parlait.

Étant scolarisés dans une école de masse, ces interviewés qualifient de «médiocres », voire de «nulles », leurs compétences linguistiques à la sortie du secondaire. De même, ils ne voyagent à l'étranger que tardivement et, pour certains, le séjour d'études en France sera leur premier voyage au-delà de la Russie.

L'anglais, j'étais zéro [au moment de la sortie du secondaire]. Je savais conjuguer le verbe to be, j'avais une certaine idée sur le système des temps, je connaissais une cinquantaine de mots. [...] Je n'ai jamais été nulle part. Mon passeport étranger, je ne l'ai fait que... [juste avant le départ étudiant en France, c'està-dire à 26-27 ans environ] [...] « Je n'irai nulle part » [me disais-je à l'époque]. J'en étais sûre, tu vois. (Sabina)

Ces interviewés évoquent souvent les contraintes matérielles qui ont marqué leur enfance : alimentation peu diversifiée, privations en matière d'objets de première nécessité et de loisirs, difficultés financières durables de leurs parents dans le contexte postsoviétique. Dans quelques cas, il s'agit d'une accumulation de plusieurs facteurs de précarité : le métier des parents de moindre qualification et/ou un emploi dans les secteurs les plus menacés durant les années 1990 (salariés de l'éducation et de la santé, petits salariés non manuels dans le secteur public ou salariés manuels), un lieu de résidence défavorable (province éloignée et/ou peu urbanisée) et une configuration familiale vulnérable (familles monoparentales ou nombreuses, parents âgés ou à la retraite).

Les parents de ces interviewés ne participent qu'à titre exceptionnel à la formation de l'idée d'étudier ailleurs qu'en Russie. Ils sont souvent ceux qui réprouvent une mobilité et dissuadent leurs enfants d'entreprendre un départ. Pour ces derniers (les cas de Véra, Polina, Anton ou Zarina, entre autres), il sera ainsi davantage question de faire accepter aux parents l'idée d'un départ, de relativiser leur opinion et, parfois, de ne plus entretenir de contacts avec eux. La mobilité de ces interviewés se conçoit clairement en rupture envers leur milieu familial, et cela est d'autant plus visible par comparaison avec le premier groupe dans lequel, au contraire, tous perpétuent un projet parental. Il s'agit de «boursiers » dans le sens de Hoggart (1970 : 347357), coupés, par leur réussite scolaire, de leur classe d'origine. De fait, on retrouve de fortes différences entre le parcours scolaire de ces interviewés et celui des membres de leur famille. 
Par exemple, Ksenia (élevée par sa mère, petite employée de bureau) et Polina (de père chauffeur et de mère infirmière) détiennent deux diplômes d'études supérieures, dont un étranger, alors que le frère de la première est plombier et celui de la seconde moniteur-ajusteur.

La conception d'une mobilité internationale d'études est clairement associée dans ces entretiens à des influences extra-familiales et postscolaires, parfois postuniversitaires. Sa réalisation dépend de façon ultime des ressources financières extérieures à la famille d'origine (bourse d'études, travail d'étudiant, contrat de fille au pair,...). Il s'agit pour plusieurs dans ce groupe d'un apprentissage culturel qu'offre le milieu universitaire et d'une ouverture d'esprit se produisant en contact avec des enseignants et/ou des pairs plus informés et plus expérimentés au niveau international. C'est le cas de Véra, par exemple, qui se qualifie d' « assez ignorante » à l'époque et découvre à l'université des univers que ni son milieu familial ni l'école ne l'ont amenée à connaître :

Je ne lisais rien, je ne regardais aucun film. Quand on me demandait si j'avais lu ou vu cela ou ceci, je ne pouvais répondre que non, non et encore non. Aucune culture, en bref. [...] [Un de mes professeurs] me donnait des disques, des livres. [...] C'est grâce à [ce professeur] que j'ai appris l'existence, tout simplement l'existence, de cette école de design [à Paris]. Je savais en principe qu'il existait une France, tout comme la Lune ou la Chine, mais... [c'était en dehors de mes investigations].

De façon systématique, une séparation s'observe dans ces cas entre les univers familial et universitaire, ce qui complique pour plusieurs leur engagement dans le départ étudiant. On constate que Véra est tiraillée entre les dires de ses parents, ouvriers de bâtiment (« oublie ça, on n'a pas d'argent ») et les paroles émanant de son enseignant d'université («Tout est possible, tu es jeune, tu as toute la vie devant toi, tu peux y aller, [...] tu dois y aller. Mets-toi à apprendre le français! »). En même temps, cette interviewée apparaît comme quelqu'un qui a une certaine ancienneté dans ce qui est de la relativisation de l'opinion de ses parents. Leur incompétence concernant ses projets d'études est soulignée plusieurs fois par la jeune femme : «choisis toimême, on n'y comprend rien », entend-elle, par exemple, de leur part, concernant son choix de filière supérieure. Ce n'est pas à l'aide de ses parents, veut nous communiquer l'interviewée, qu'elle a géré son cursus universitaire, national comme international. Une même autonomie précoce envers la famille d'origine s'observe dans d'autres cas, dont celui d'Anton ou de Ksenia.

Outre les enseignants, des amis et des connaissances peuvent faire songer à un voyage d'études à l'étranger et contribuer ainsi à concevoir une mobilité internationale. C'est le cas de Sabina, entre autres, qui abandonne sa représentation de longue date («je n'irai nulle part ») suite à la conversation avec une amie de l'université tout juste reçue à un concours pour une bourse d'études dans un établissement français. Les pairs qui jouent un rôle dans la conception d'une mobilité sont présentés dans ces entretiens comme plus expérimentés et plus compétents que les interviewés eux-mêmes en matière de circulation internationale. Ils disposent fréquemment de plus de ressources pour effectuer une mobilité d'études. Par exemple, « la meilleure amie » d'université de Ksenia est une ancienne élève de l'école renforcée en anglais et, deux années avant le départ de Ksenia en France, elle va étudier le marketing à Londres, délivrant ainsi un modèle à suivre pour l'interviewée. De même, le départ de Polina doit beaucoup à la relation d'amitié, durant ses études universitaires, avec « une fille de parents riches et avancés, célèbres sportifs, qui voyageait beaucoup à l'étranger depuis l'enfance », pour reprendre les propos de l'interviewée.

Â cet égard, 1'hétérogénéité des réseaux de sociabilité semble augmenter la probabilité d'être incité à effectuer un séjour d'études à l'étranger. En même temps, beaucoup d'interviewés se révèlent fréquenter des milieux plutôt homogènes où certaines choses se pratiquent ou se disent, et d'autres non. La fréquence d'expressions de type «tous mes amis pensent ceci ou cela » confirme ce point. La possibilité de côtoyer le milieu de pairs où l'on peut être encouragé à partir à l'étranger apparaît alors assez réduite pour certains (école de masse, filière supérieure peu internationalisée). C'est en effet une rupture préalable avec l'entourage habituel qui leur ouvre la possibilité de nouer des relations avec quelqu'un pouvant conduire à un départ étudiant. De cette façon, avant d'être incités à une mobilité, plusieurs interviewés entreprennent des démarches allant à l'encontre de ce qui se fait dans leur milieu et s'entourent de personnes qu'ils n'ont pas fréquentées jusqu'alors. Issus de familles peu diplômées, ils parviennent à entrer à 
l'université nationale (Polina, Ksenia, Véra ou Tamara) et, notamment, à intégrer une filière supérieure fortement compétitive qui ne leur était pas «promue » (Zarina); ils envisagent un changement de métier et font des connaissances dans des mondes jadis inconnus (Ivan) ; préoccupés par des lacunes dans leur éducation, ils vont étudier des langues à l'âge adulte (Yulia ou Sofia), ou bien, étant jusqu'alors de faibles voyageurs, entreprennent un déplacement et nouent dès lors des relations aussi bien décisives qu'inédites (Mila). C'est ainsi que, par l'intermédiaire de ces nouveaux amis et connaissances que ces interviewés sont amenés à côtoyer lors d'activités toutes nouvelles, une incitation ou une série d'incitations envers un départ à l'étranger émanent.

Notons, en outre, que les interviewés non mobiles peuvent également utiliser les locutions «toujours » et « jamais » pour parler de la mobilité d'études. Certains parmi les non mobiles anticipent en effet ma question concernant d'éventuelles études à l'étranger, préparée pour la fin de l'entretien : ils en parlent dès le début de la conversation, et ce pour dire qu'ils y pensent « depuis toujours ». Tous anciens élèves d'écoles sélectives et issus de familles aisées, ils montrent, durant l'entretien, avoir bien conçu une mobilité ou être en train de préparer leur départ. En revanche, dans d'autres entretiens avec les non mobiles, ma question concernant un voyage d'études est perçue comme déplacée ; elle fait rire ou rend perplexe : « Moi ? À l'étranger ? [il rit] Bah, cela ne m'est jamais venu à l'esprit ! » (Stépan). En examinant les modalités de la socialisation familiale et scolaire de ces interviewés, on constate que ce type de réponse correspond à des scolarisations peu compétitives et à des conditions d'origine très modestes.

\subsection{Une mobilité convoitée mais d'une légitimité variable}

Un autre axe de variation que la temporalité apparaît dans les entretiens. Les propos recueillis varient notamment concernant la légitimité que les interviewés attribuent à leur souhait d'aller étudier à l'étranger. Si, pour les uns, ce souhait est «normal » (Natalia), «naturel » (Diana) ou « logique » (Konstantin), pour d'autres, il a constitué, pendant plus ou moins longtemps, un 《rêve irréaliste » (Polina), une idée « osée » (Ksenia), « interdite » (Véra) ou « folle » (Irina). Ces variations n'apparaissent pas non plus socialement neutres, mais en lien avec les conditions d'origine et le parcours scolaire des personnes rencontrées.

Ainsi, pour une partie des interviewés, leur conception de la mobilité est comparable à celle de jeunes Européens issus des classes moyenne et supérieure pour qui il est «normal » d'envisager une expérience internationale : " c'est dans la logique des choses », comme l'explique une enquêtée mobile de Cicchelli (2012 : 47). Par exemple, Andrey Z., ancien élève d'une école anglaise et doctorant d'AgroParisTech au moment de l'entretien, n'hésite pas à se proposer plusieurs projets de circulation internationale quant à ses études, ses loisirs ou son travail. Il en parle avec une familiarité notable:

Je n'étais pas d'emblée convaincu par la France, j'avais plutôt l'Autriche en tête. C'était comme ça, historiquement. À l'université, par exemple, je suis allé en Autriche pour quelques mois. Mon père travaillait par ailleurs là-bas à l'époque [avec une mission diplomatique]. [...] [Après mes études à la faculté de management international à Saint-Pétersbourg,] je cherchais un travail et je suis tombé sur une annonce d'une compagnie autrichienne. Je leur ai envoyé mon CV, ils m'ont appelé. Ils voulaient me faire venir à Moscou pour passer un entretien. Mais puisque justement, à ce moment-là, $j$ 'allais en Autriche pour faire du ski, je leur ai proposé de nous retrouver directement à Vienne. Je suis donc venu, tranquillement. On a parlé un peu et ils m'ont embauché. [...] Ils voulaient quelqu'un maîtrisant l'anglais, l'allemand et le russe, et c'était mon cas. Plus un diplôme convenable, en business international ou management. En bref, naturellement, j'étais dans l'optique de l'Autriche jusqu'à mes 25 ans environ. Ce n'est que par la suite... En effet, c'est quand j'étais en Amérique du Sud, avec une copine slovaque, que... C'est là-bas que j'ai pensé à d'autres destinations : l'Italie pour étudier l'art ou la Suède pour obtenir un master intitulé Road to Democracy [petit rire]. [...] [Lors du travail en Autriche,] je cherchais des programmes d'études et j'ai trouvé celui-ci à Paris [à HEC]. Initialement donc, je ne pensais pas aller en France. Pour autant, je me suis rendu dans ce pays deux fois. Comme touriste d'abord, puis pour participer à un business game pour les étudiants [de différents pays], organisé par L'Oréal. 
On comprend par ailleurs que la non mobilité des interviewés au profil comparable n'est pas due à des difficultés à concevoir un voyage d'études, mais plutôt à celles de préparer le départ. Notamment, pour plusieurs, leur préparation du départ inflige des sentiments douloureux liés à un certain anéantissement des espérances associées au parcours antérieur (scolarité privilégiée, compétences linguistiques coûteuses à acquérir, expériences internationales antérieures relativement rares,...). C'est parfois au prix d'une revue à la baisse des exigences et des attentes que leur départ se réalise : étudier en France alors que l'on voudrait se rendre en Angleterre (Élisaveta et Kristina), en province au lieu de Paris (Diana et Rada), s'inscrire à la faculté alors que l'on visait une école (Varvara et Olga D.), partir sans encadrement institutionnel ni bourse (Varvara et Diana). Tout en se considérant être en plein droit de circuler à l'international, ils sont amenés à découvrir que la réalisation d'un départ étudiant ne va pas de soi.

Dans d'autres entretiens, on observe, au contraire, des restrictions que les interviewés imposent à leur désir d'aller à l'étranger. Ils qualifient ce dernier d'utopique et ne s'autorisent pas à y penser. Tout se passe comme s'ils souhaitaient désespérément ce qui leur était refusé, pour paraphraser la formule célèbre de Bourdieu concernant « cette sorte de soumission immédiate à l'ordre qui incline à faire de nécessité vertu, c'est-à-dire à refuser le refusé et à vouloir l'inévitable » (1980:90).

Quand j'étais en Russie, je pensais que les études à l'étranger étaient réservées aux enfants de parents très très riches, ou aux enfants très talentueux. Je ne me considérais comme ni l'une ni l'autre [petit rire]. [...] Je savais qu'à l'étranger tout a un prix, et j'avais l'impression que mes parents n'avaient pas assez d'argent pour payer mes études ni pour louer un appartement, toutes ces choses qui coûtent cher. Concernant le talent... Je n'étais pas la première de ma classe. Enfin si, j'étais parmi les premiers, mais... cela ne veut rien dire. À l'université, j'étais moyenne. Je n'étais pas «très bien », j'étais «bien». Or, les bourses, par exemple, sont versées aux meilleurs des meilleurs. [...] [Comme] je suis assez réaliste, je ne me permettais pas de rêver à ce sujet. (Diamara)

J'avais cet état esprit, j'avais cette petite pensée [d'étudier à l'étranger] bien avant de... [mes études universitaires] Mais... je n'osais pas rêver ! Je ne suis pas géniale, c'est évident. J'ai terminé l'école sans mention. Et... en réalité, je n'étais pas sûre d'avoir le droit d'y penser. (Tamara)

Cette représentation nommée « réaliste » s'acquiert en famille et à l'école. Si les expériences familiale et scolaire d'Andrey Z. présentent un apprentissage prolongé de possibilités d'une mobilité internationale, celles d'autres interviewés font, au contraire, état d'un processus lors duquel ils apprennent que circuler à l'international ne leur est pas, selon l'expression de Polina, «prédestiné »:

Rien n'annonçait ce scénario [de mobilité]. Je suis née en province, à 450 kilomètres de Moscou, dans une ville de 100000 habitants. Tu comprends ce que cela veut dire en termes d'essor de la pensée? [petit rire] Ma famille est absolument prolétarienne, cela ne me prédestinait pas non plus à partir en France. Comme mon papa m'a dit : «Quelle France encore ? ! D’où cette idée t'est-elle venue ? La France, c'est pour les enfants des riches et pour les prostituées!»

Au-delà de la connotation négative de la mobilité internationale relevée chez certains parents, les interviewés qui considèrent comme illégitime leur souhait, pourtant présent depuis longtemps, d'aller étudier à l'étranger, ont d'autres d'occasions de confirmer cette représentation. Par exemple, l'expérience scolaire, puis universitaire de plusieurs est marquée par des comparaisons amères avec leurs camarades plus aisés et/ou plus performants en études, ainsi que par des restrictions des dépenses financières liées à leur éducation et par des renoncements divers. Une série de résignations s'observe dans le cas d'Élena C. : au projet d'étudier à Saint-Pétersbourg («je n'étais pas si sûre de moi », dit-elle), à celui d'entrer à la faculté de la langue anglaise d'une ville de province (« le concours était ardu ; on m'avait d'ailleurs dit que, sans argent ni relations, ce serait impossible [d'y entrer] ») et à celui de devenir traductrice-interprète du français ( je comprenais qu'au mieux je deviendrais institutrice d'école ou secrétaire »). Ces expériences aboutissent chez plusieurs à la formation d'un jugement très modeste qu'ils portent sur eux-mêmes et à des blocages pour concevoir une mobilité internationale d'études en tant qu'avenir légitime. Cela se confirme par plusieurs entretiens avec les non mobiles, comme avec Marina P. qui «aimerai[t] bien » étudier à l'étranger, mais qui considère cela comme « une ambition stratosphérique » qu'elle n'est pas « capable » de réaliser. 
Un contre-apprentissage doit ainsi avoir lieu pour ces interviewés afin qu'ils évacuent leurs doutes et pensent plus aisément à la mobilité internationale. L'examen de plusieurs récits révèle une certaine reconversion mentale de ces interviewés. Lors de cette dernière, ils vivent une véritable démystification d'un départ pour les études à l'étranger - qu'ils jugeaient jusqu'alors comme un comportement de luxe réservé à une minorité de personnes fortunées et/ou dotées de « talents » innés (pour les langues ou pour d'autres matières). De même, ils prennent progressivement confiance en eux et en leur capacité à agir.

On constate, par exemple, que, durant les études universitaires nationales de Tamara, la représentation de cette dernière sur la mobilité internationale se modifie. Ainsi, son souhait d'aller étudier ailleurs ne lui paraît plus déplacé, et elle est amenée à reconnaître une banalité des voyages d'études à l'étranger. Ce processus se déroule, bien sûr, en interaction avec d'autres personnes. C'est en fréquentant des milieux plus diversifiés que ceux de sa ville d'origine, d'abord à Ekaterinbourg, puis à Saint-Pétersbourg, que la jeune femme est conduite à redéfinir son souhait comme réalisable. Ce ne sont pourtant pas seulement, ni principalement, des incitations reçues de l'extérieur qui comptent. En effet, entrer à l'université nationale représente déjà pour l'interviewée une action forte et inédite qui constitue une sorte de première marche en direction de son départ étudiant à l'étranger. Ayant réussi le concours d'entrée, la jeune femme apprend qu'elle peut réaliser ce que son entourage, celui d'une «petite ville ouvrière » où «tous deviennent ouvriers », considère comme utopique. Lors de ses études à Ekaterinbourg, Tamara envisage un déménagement à Saint-Pétersbourg et réussit son transfert universitaire. La prise de conscience de ses capacités (elle comprend qu'elle « peut »: "vivre autrement », « changer ») vient comme une conséquence de ses propres actions. Or, ce n'est pas en restant à la maison que l'interviewée comprend « réellement» que les choses sont faisables, mais en prenant l'initiative de les faire. Petit à petit, en s'assurant que les actions dont le succès lui paraissait incertain sont à sa portée, elle avance envers la reconnaissance de la faisabilité d'un départ d'études à l'étranger. Chacune de ses réussites (études supérieures, transfert à SaintPétersbourg, entrée en doctorat) lui autorise de plus grands espoirs.

Une même régularité s'observe concernant l'étude d'une langue étrangère. Apprendre une langue étrangère est en effet perçu comme une tâche d'emblée écrasante par beaucoup d'interviewés, raison pour laquelle ils hésitent à envisager une mobilité :

Je me disais [à l'époque] : «Non, non, c'est impossible. Je ne me souviens plus depuis quand j'étudie cet anglais chiant, mais je suis toujours loin de la perfection. Je ne réussirai jamais avec le français, c'est impossible. » (Zlata)

Progressivement, une maîtrise se construit pourtant : Zlata se met à étudier la langue française, d'abord en autodidacte, depuis «les choses les plus élémentaires ». Elle avoue avoir confondu les verbes «être » et «avoir», souffre de sa «mauvaise mémoire » et constate ne pas être « douée » en langues. Grâce à un travail acharné de quelques années, l'interviewée avance pour autant vers le niveau $\mathrm{C} 1$ qui lui est nécessaire pour entrer à l'École du Louvre : ce qui était jadis « impossible » pour elle s'avère faisable à travers ses propres actions.

\section{Conclusion}

En conclusion, les compétences internationales acquises durant la scolarisation dans le pays d'origine sont variables chez les Russes interviewés. Plusieurs facteurs se conjuguent sur ce plan, et notamment les ressources socio-économiques et culturelles inégales de leur famille d'origine. Par conséquent, ce n'est ni dans la même temporalité ni avec une même légitimité que les interviewés envisagent une mobilité internationale, et la comparaison entre les mobiles et les non mobiles confirme ce constat. Si les uns apparaissent précocement confortés dans la perspective internationale, voire programmés pour celle-ci, pour d'autres, étudier à l'étranger renvoie pendant longtemps au domaine de l'impossible, et ils sont contraints de modifier leurs représentations : grâce à des interventions extérieures, ainsi qu'à travers leurs propres actions qui les amènent à rompre avec leur milieu d'origine. En poursuivant l'analyse, on peut avancer que plusieurs variations apparaissent chez les interviewés durant la phase de la préparation du départ. Notamment, la durée de cette phase fluctue selon leurs ressources financières et compétences linguistiques initiales, allant de quelques mois pour certains à plusieurs années 
pour d'autres. De même, le choix du pays d'études, qui se précise à cette phase, peut être limité pour les uns à un seul pays, comme la France, et à des filières peu sélectives. Ce n'est pas le cas pour d'autres interviewés dont les compétences linguistiques ne se résument pas à une seule langue étrangère maîtrisée et qui disposent des moyens économiques leur permettant d'envisager les prix élevés d'études. La distinction qui s'opère ainsi au sein même des parcours internationaux de formation trouve une partie de son origine dans l'accès inégal initial aux espaces éducatifs nationaux.

\section{Bibliographie}

Ballatore M. (2010), Erasmus et la mobilité des jeunes Européens, Paris, Presses universitaires de France.

BeCKer H. (1985), Outsiders. Études de sociologie de la déviance, Paris, Métailié.

BerELOWITCH W. (2000), «École et enseignement : la fin d'un rêve », Transitions, n 2, p. 51 60.

Bourdieu P. (1980), Le sens pratique, Paris, Minuit.

Bourdieu P., PASSERON J.-C. (1964), Les héritiers. Les étudiants et la culture, Paris, Minuit.

CiCCHELli V. (2012), L'esprit cosmopolite. Voyages de formation des jeunes en Europe, Paris, Presses de Sciences Po.

DARMON M. (2008), Devenir anorexique. Une approche sociologique, Paris, La Découverte.

DARMON M. (2012), La socialisation, Paris, Armand Colin.

Duru-Bellat M., JarléGAN A. (2001), «Garçons et filles à l'école primaire et dans le secondaire », in T. BLÖSS (dir.), La dialectique des rapports hommes-femmes, Paris, Presses universitaires de France, p. 73-88.

ENDRIZZI L. (2010), «La mobilité étudiante, entre mythe et réalité », Dossier d'actualité de la $V S T, \mathrm{n}^{\circ} 51$.

GARNEAU S. (2008), « Inégalités d'accès à l'espace international de l'enseignement supérieur et aux marchés du travail », in L'enseignement supérieur dans la mondialisation libérale. Une comparaison internationale (Maghreb, Afrique, Canada et France), Tunis, IRMC, p. 133-150.

GÉRARD É. (dir.) (2008), Mobilités étudiantes Sud-Nord. Trajectoires scolaires de Marocains en France et insertion professionnelle au Maroc, Paris, Publisud.

HoggarT R. (1970), La culture du pauvre, Paris, Minuit.

MARKosyan A. (2000), «Situation de la langue française en Russie : le français, deuxième langue étrangère », Cahiers de l'Association internationale des études françaises, $\mathrm{n}^{\circ}$ 52, p. 3139.

REY M.-P. (dir.) (2005), Les Russes de Gorbatchev à Poutine, Paris, Armand Colin.

TCHEREDNITCHENKO G. (1993), «Un espace réservé. Les écoles spéciales de langues étrangères en Union Soviétique », Actes de la recherche en sciences sociales, vol. 98, p. 63-68.

TCHEREDNitCHENKo G., LAPPARENT D. DE (2000), «L'école secondaire russe en mutation (d'après la situation à Moscou) », Revue d'études comparatives Est-Ouest, vol. 31 n 3, p. 99125. 
VINOKUR A. (dir.) (2001), Les transformations du système éducatif de la fédération de Russie, Paris, UNESCO.

WAGNER A.-C. (2007), Les classes sociales dans la mondialisation, Paris, La Découverte.

YAN A. (2009), «Les étudiants chinois dans les grandes écoles. Une vision de l'excellence », in C. Agulhon, A. XAVIER De Brito (dir.), Les étudiants étrangers à Paris. Entre affiliation et repli, Paris, L'Harmattan, p. 157-202. 